\title{
Clinical outcomes after early ambulatory multidrug therapy for high-risk SARS-CoV-2 (COVID-19) infection
}

\author{
Brian C. Procter ${ }^{1}$, Casey Ross ${ }^{1}$, Vanessa Pickard ${ }^{1}$, Erica Smith ${ }^{1}$, Cortney Hanson ${ }^{1}$ and Peter A. McCullough ${ }^{2,3,4, *}$ \\ ${ }^{1}$ McKinney Family Medicine, McKinney, 75070, TX, USA \\ ${ }^{2}$ Baylor University Medical Center, Dallas, 75226, TX, USA \\ ${ }^{3}$ Baylor Heart and Vascular Institute, Dallas, 75226, TX, USA \\ ${ }^{4}$ Baylor Jack and Jane Hamilton Heart and Vascular Hospital, Dallas, 75226, TX, USA \\ ${ }^{*}$ Correspondence: peteramccullough@gmail.com (Peter A. McCullough)
}

DOI: $10.31083 /$ i.r.rm.2020.04.260

This is an open access article under the CC BY 4.0 license (https://creativecommons.org/licenses/by/4.0/).

There is an emergency need for early ambulatory treatment of Coronavirus Disease 2019 (COVID-19) in acutely ill patients in an attempt to reduce disease progression and the risks of hospitalization and death. Such management should be applied in high-risk patients age > 50 years or with one or more medical problems including cardiovascular disease. We evaluated a total of 922 outpatients from March to September 2020. All patients underwent contemporary real-time polymerase chain reaction (PCR) assay tests from anterior nasal swab samples. Patients age $50.5 \pm 13.7$ years (range 12 to 89 ), $61.6 \%$ women, at moderate or high risk for COVID-19 received empiric management via telemedicine. At least two agents with antiviral activity against SARS-CoV-2 (zinc, hydroxychloroquine, ivermectin) and one antibiotic (azithromycin, doxycycline, ceftriaxone) were used along with inhaled budesonide and/or intramuscular dexamethasone consistent with the emergent science on early COVID-19 treatment. For patients with high severity of symptoms, urgent in-clinic administration of albuterol nebulizer, inhaled budesonide, and intravenous volume expansion with supplemental parenteral thiamine $500 \mathrm{mg}$, magnesium sulfate 4 grams, folic acid 1 gram, vitamin B12 $1 \mathrm{mg}$. A total of $320 / 922(34.7 \%)$ were treated resulting in $6 / 320$ $(1.9 \%)$ and $1 / 320(0.3 \%)$ patients that were hospitalized and died, respectively. We conclude that early ambulatory (not hospitalized, treated at home), multidrug therapy is safe, feasible, and associated with low rates of hospitalization and death. Early treatment should be considered for high-risk patients as an emergency measure while we await randomized trials and guidelines for ambulatory management.

\section{Keywords}

SARS-CoV-2; COVID-19; multidrug; hospitalization; mortality; ambulatory; antiviral; zinc; hydroxychloroquine; ivermectin; doxycycline; azithromycin; vitamin; corticosteroid

\section{Background}

The epidemic viral outbreak of Severe Acute Respiratory Syndrome Coronavirus 2 (SARS-CoV-2) infection Coronavirus Disease 2019 (COVID-19) is advancing across the United States unabated despite public policy measures focussed on contagion control (McCullough et al., 2020). The United States has a current 877 deaths per million inhabitants despite having technically advanced hospitals and to date sufficient capacity to handle the surges of patients requiring hospitalization (Worldometer, 2020). Conversely India, a country with broad implementation of early COVID-19 treatment has 102 deaths per million (Worldometer, 2020). The regulatory agencies as well as the National Institutes of Health have had their principal areas of focus being late stage hospitalized patients and vaccine development (COVID-19 Treatment Guidelines, 2020). This has left a void for the role of early ambulatory treatment of COVID-19 at home. Such management has the goals of lessening the intensity and severity of symptoms and preventing hospitalization and death. There are currently no approved drugs or drug combinations in the U.S. indicated for the ambulatory treatment of COVID-19 or its complications. In the absence of conclusive randomized trials of single drugs and combination regimens, clinicians faced with large numbers of ill patients have responded with innovative empiric approaches that attempt to reduce the progression of SARS-CoV-2 infection, improve symptoms, avoid complications, and reduce the risk of complications and death. The mechanisms by which a multidrug approach would globally improve outcomes could be to address viral replication, cytokine storm, and thrombosis. This report discloses real world data and the clinical outcomes of early ambulatory treatment of acute COVID-19 in patients at high risk for hospitalization and death.

\section{Methods and results}

Beginning in March 2020, a team of primary care providers consisting of a lead physician (BCP) and four advanced practice practitioners (CR, VP, ES, $\mathrm{CH}$ ) responded to urgent visits by patients with suspected SARS-Co-V infection and symptomatic COVID-19. All patients underwent standard informed consent for 

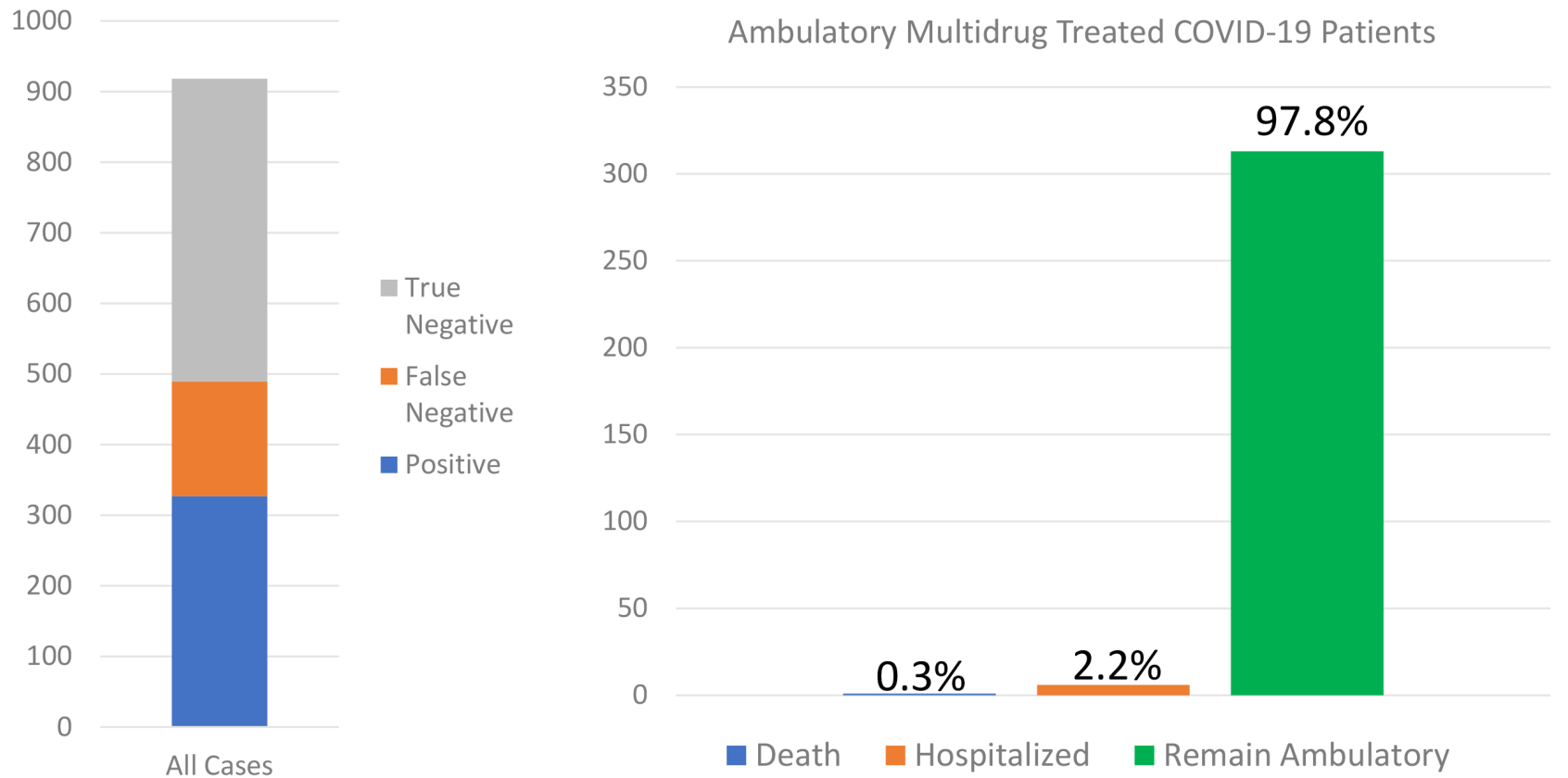

Fig. 1. The SARS-CoV-2 nasal PCR test results are shown on the left and among the 320 cases that were confirmed positive and were high risk, the outcomes of hospitalization and death are shown on the right.

Table 1. Symptom severity score for initial assessment of patients infected with suspected infection of SARS-CoV2 (COVID-19).

\begin{tabular}{lc}
\hline Symptom & Points \\
\hline Fever & 1 \\
Fever at night & 1 \\
Fatigue & 1 \\
Body aches & 1 \\
Cough & 1 \\
Difficulty breathing & 1 \\
Additional symptoms & 1 \\
Probability of COVID-19: $0-1$ points = low, 3-4 points = moder- & \\
ate, 5+ points = high & \\
\hline
\end{tabular}

care and were under the direct management of licensed medical personnel including a senior attending physician (BCP). Contemporary real-time polymerase chain reaction (PCR) assay tests from anterior nasal swab samples were obtained. It was understood at that time period that COVID-19 test results could be falsely negative, particularly in the setting where a patient had the characteristic symptoms of the syndrome (Woloshin et al., 2020). They additionally had an assessment according to the severity of symptoms and scored as depicted in Table 1. The treatment regimens are given in Table 2. All patients received empiric treatment on the first day of presentation in most cases before COVID-19 test results with standard office practice and contagion control measures (Fiorillo et al., 2020). According to clinical judgment and planned use of hydroxychloroquine (HCQ) a 12-lead electrocardiogram was obtained to evaluate the QTc interval. For patients with high severity of symptoms, urgent in-clinic administration of albuterol nebulizer, inhaled budesonide, and intravenous volume expansion with supplemental parenteral thiamine $500 \mathrm{mg}$, magnesium sulfate 4 grams, folic acid 1 gram, vitamin B12 $1 \mathrm{mg}$ (Flannery et al., 2017). Additionally, for the severely ill population dexamethasone $8 \mathrm{mg}$ and ceftriaxone 1 gram was administered intramuscularly (Table 2). All patients had in-person or telemedicine followup at 48 hours and as needed after that point which was part of the general consent for treatment. Univariate statistics were reported with means \pm standard deviation or counts with proportions as appropriate.

A total of 922 patients were evaluated between the ages of 12 and 89 years. The mean age was $50.5 \pm 13.7$ years and $61.6 \%$ were women. The frequency of comorbidities was as follows: obesity $60.5 \%$, diabetes mellitus $10 \%$, cardiovascular disease $33.7 \%$, pulmonary illness $17.8 \%$. The rate of positive SARS-CoV-2 positive tests was 327/918 (35.6\%). Among the 591 test negative patients, $162(27.4 \%)$ were considered false negative tests since they went on to develop persistent or worsening symptoms of COVID-19. A total of 320/922 (34.2\%) were treated based on age $>50$ and/or the presence of comorbidities (obesity, diabetes mellitus, cardiopulmonary disease, chronic kidney disease, etc). All patients were followed for a minimum of 90 days. Clinical outcomes included $6 / 320(1.9 \%)$ and $1 / 320(0.3 \%)$ that were hospitalized or died, respectively (Fig. 1).

\section{Discussion}

The observations in this report suggest that primary care physicians can take an organized, empiric approach to acutely ill patients with COVID-19 with very low rates of subsequent hospitalization and death. The execution of this program was heavily dependent on telemedicine technology (Cervino and Oteri, 2020). Our observations suggest a majority of hospitalizations could be 
Table 2. Combination medications for a minimum of five days and acutely administered supplements used for the initial ambulatory patient with suspected and or confirmed COVID-19 (moderate or greater probability).

\begin{tabular}{ll}
\hline Agent & Rationale \\
\hline Zinc & Inhibits SARS-CoV-2 RNA synthesis \\
Hydroxychloroquine $200 \mathrm{mg}$ po bid & Inhibits endosomal transfer of virions, anti-inflammatory \\
Ivermectin $(200 \mathrm{mcg} / \mathrm{kg})$ usual dose $12 \mathrm{mg}$ po qd $\times 3$ days & Attenuates importin $\alpha / \beta$-mediated nuclear transport of SARS-CoV-2 into nucleus \\
Azithromycin $250 \mathrm{mg}$ po bid & Covers respiratory bacterial pathogens in secondary infection \\
Doxycycline $100 \mathrm{mg}$ po bid & Covers respiratory bacterial pathogens in secondary infection \\
Inhaled budesonide, Dexamethasone $8 \mathrm{mg} \mathrm{IM}$ & Treats cytokine storm \\
Folate, thiamine, vitamin 12 & Reduce tissue oxidative stress \\
Intravenous fluid & Intravascular volume expansion \\
\hline
\end{tabular}

avoided and the spread of SARS-CoV-2 can be reduced with a first treat-at-home approach featuring telemedicine during followup (Gambardella et al., 2020; Tolone et al., 2020). We leveraged of agents that were commercially available and had a reasonable chance of therapeutic gain with acceptable safety. Because multiple agents are used empirically and in combination given the context of an emergency pandemic, it is impossible to retrospectively stratify for each component and analyze individual effects. We addressed viral replication, cytokine storm, and tissue damage due to oxidative stress utilizing vitamins, micronutrient supplements, and prescription medications (Zhang et al., 2020). Additionally, we encouraged the use of renin-angiotensin system inhibitors based on their theoretical effect over the long term for upregulation of the angiotensin converting enzyme 2 receptor which, despite being the entry receptor for the SARS-CoV-2 also protects lungs in preclinical models of adult respiratory distress syndrome (Lo et al., 2020; Palazzuoli et al., 2020). Our approach was later supported by concurrent analyses and subsequent published reports (Derwand et al., 2020; Lo et al., 2020; Palazzuoli et al., 2020). The observed rates of these outcomes are considerably lower than reported in other studies in our region. A recent report from Methodist hospital in Houston reported that patients with progressive symptoms when hospitalized suffered a $5.8 \%$ mortality rate was despite the use of HCQ, remdesivir, convalescent plasma, and anticoagulants (Vahidy et al., 2020). Undoubtedly a portion of the mortality benefit of outpatient therapy is reducing the need for supplemental oxygen and mechanical ventilation. A recent series from Italy has demonstrated that there is a graded increase in death rates with $7.4 \%$ for whom no oxygen was required, $12.9 \%$ for oxygenrequiring, and $23.0 \%$ for mechanically ventilated patients (Palazzuoli et al., 2020). Our data suggest the advancement of early home use of off-target antiviral agents (zinc, HCQ, ivermectin, azithromycin, doxycycline), antibiotics, corticosteroids, and in the future empiric anticoagulants could markedly reduce the risk for hospitalization and potentially reduce overall death rates before and during hospitalization (McCullough et al., 2020).

Our report has all the limitations common to the reporting of clinical practice outcomes. During this time there was an evolving set of SARS-CoV-2 assays, and hence when assessed in context to the clinical syndrome, we experienced both false positive and negative testing as reported. Follow-up was performed by usual practice call logs and electronic medical systems and is temporally truncated to the time of this report.

\section{Conclusions}

In conclusion, empiric multidrug treatment for ambulatory COVID-19 according to age, comorbidities, and initial severity of symptoms is feasible with close follow-up. Our data suggest that such a strategy is associated very low rates of hospitalization in high-risk patients who receive early outpatient treatment. This may be due to symptom relief attributed to medications, supportive parenteral volume expansion, micronutrient supplementation, and compassionate care delivered by in person visits and telemedicine. The rates of death in our study indicate that early multidrug therapy is associated with $>90 \%$ reduction in mortality among the high risk compared to community rates of death associated with therapeutic nihilism in ambulatory patients who are subsequently hospitalized. The National Institutes of Health currently advise denial of early treatment and encourage late-stage hospitalization as the first window of treatment open to acutely ill patients with COVID19 (COVID-19 Treatment Guidelines, 2020). Our contrary view, supported by our results, is that early ambulatory therapy should be offered as an emergency measure in acutely ill, high-risk COVID19 as a strategy to reduce hospitalization and death. We anticipate results of clinical trials will refine our multipronged therapeutic response to patients with this potentially fatal infection, however in our view, there is an impressive and urgent call for action at the earliest point in the infection for the best chances of survival from hospitalization and death.

\section{Authors' contributions}

BCP, CR, VP, ES, CH, contributed patient data, PAM drafted the first version, and all authors contributed edits to the final version.

\section{Ethics approval and consent to participate}

All patients provided informed consent for treatment according to good clinical practice.

\section{Acknowledgments}

There are no acknowledgments to disclose.

\section{Conflict of interest}

Nothing to disclose. Authors had access to the data and wrote the manuscript. 
Submitted: November 26, 2020

Revised: December 09, 2020

Accepted: December 14, 2020

Published: December 30, 2020

\section{References}

Cervino, G. and Oteri, G. (2020) COVID-19 pandemic and telephone triage before attending medical office: problem or opportunity? Medicina (Kaunas) 56, 250.

COVID-19 Treatment Guidelines. (2020) Available at: https://www.co vid19treatmentguidelines.nih.gov/ (Released: October 8, 2020).

Derwand, R., Scholz, M. and Zelenko, V. (2020) COVID-19 outpatients: early risk-stratified treatment with zinc plus low-dose hydroxychloroquine and azithromycin: a retrospective case series study. International Journal of Antimicrobial Agents 56, 106214.

Fiorillo, L., Cervino, G., Matarese, M., D’Amico, C., Surace, G., Paduano, V., Fiorillo, M. T., Moschella, A., La Bruna, A., Romano, G. L., Laudicella, R., Baldari, S. and Cicciù, M. (2020) COVID-19 surface persistence: a recent data summary and its importance for medical and dental settings. International Journal of Environmental Research and Public Health 17, 3132.

Flannery, A. H., Adkins, D. A. and Cook, A. M. (2017) Unpeeling the Evidence for the Banana Bag: Evidence-Based Recommendations for the Management of Alcohol-Associated Vitamin and Electrolyte Deficiencies in the ICU. Critical Care Medicine 44, 1545-1552.

Gambardella, C., Pagliuca, R., Pomilla, G. and Gambardella, A. (2020) COVID-19 risk contagion: organization and procedures in a South Italy geriatric oncology ward. Journal of Geriatric Oncology 11, 11871188.

Lo, K. B., Bhargav, R., Salacup, G., Pelayo, J., Albano, J., McCullough, P. A. and Rangaswami, J. (2020) Angiotensin converting enzyme inhibitors and angiotensin II receptor blockers and outcomes in patients with COVID-19: a systematic review and meta-analysis. Expert Review of Cardiovascular Therapy 1-12.

Lo, K. B., McCullough, P. A. and Rangaswami, J. (2020) Antihypertensive drugs and risk of COVID-19? The Lancet Respiratory Medicine 8, e29.

McCullough, P. A., Eidt, J., Rangaswami, J., Lerma, E., Tumlin, J., Wheelan, K., Katz, N., Lepor, N. E., Vijay, K., Soman, S., Singh, B., McCullough, S. P., McCullough, H. B., Palazzuoli, A., Ruocco, G. M. and Ronco, C. (2020) Urgent need for individual mobile phone and institutional reporting of at home, hospitalized, and intensive care unit cases of SARS-CoV-2 (COVID-19) infection. Reviews in Cardiovascular Medicine 21, 1-7.

McCullough, P. A., Kelly, R.J., Ruocco, G., Lerma, E., Tumlin, J., Whee- lan, K. R., Katz, N., Lepor, N. E., Vijay, K., Carter, H., Singh, B., McCullough, S. P., Bhambi, B. K., Palazzuoli, A., De Ferrari, G. M., Milligan, G. P., Safder, T., Tecson, K. M., Wang, D. D., McKinnon, J. E., O'Neill, W. W., Zervos, M. and Risch, H. A. (2020) Pathophysiological basis and rationale for early outpatient treatment of SARS-CoV2 (COVID-19) infection. The American Journal of Medicine S00029343(20)30673-2. (Online ahead of print)

Palazzuoli, A., Mancone, M., De Ferrari, G. M., Forleo, G., Secco, G. G., Ruocco, G. M., D'Ascenzo, F., Monticone, S., Paggi, A., Vicenzi, M., Palazzo, A. G., Landolina, M., Taravelli, E., Tavazzi, G., Blasi, F., Infusino, F., Fedele, F., De Rosa, F. G., Emmett, M., Schussler, J. M., Tecson, K. M. and McCullough, P. A. (2020) Antecedent administration of angiotensin-converting enzyme inhibitors or angiotensin II receptor antagonists and survival after hospitalization for COVID-19 syndrome. Journal of the American Heart Association 9, e017364.

Palazzuoli, A., Ruberto, F., De Ferrari, G. M., Forleo, G., Secco, G. G., Ruocco, G. M., D'Ascenzo, F., Mojoli, F., Monticone, S., Paggi, A., Vicenzi, M., Corcione, S., Palazzo, A. G., Landolina, M., Taravelli, E., Tavazzi, G., Blasi, F., Mancone, M., Birtolo, L. I., Alessandri, F., Infusino, F., Pugliese, F., Fedele, F., De Rosa, F. G., Emmett, M., Schussler, J. M., McCullough, P. A. and Tecson, K. M. (2020) Inpatient mortality according to level of respiratory support received for severe acute respiratory syndrome coronavirus 2 (coronavirus disease 2019) infection: a prospective multicenter study. Critical Care Explorations 2, e0220.

Tolone, S., Gambardella, C., Brusciano, L., Del Genio, G., Lucido, F. S. and Docimo, L. (2020) Telephonic triage before surgical ward admission and telemedicine during COVID-19 outbreak in Italy. Effective and easy procedures to reduce in-hospital positivity. International Journal of Surgery 78, 123-125.

Vahidy, F. S., Drews, A. L., Masud, F. N., Schwartz, R. L., Askary, B. B., Boom, M. L. and, Phillips, R. A. (2020) Characteristics and outcomes of COVID-19 patients during initial peak and resurgence in the Houston metropolitan area. Journal of the American Medical Association 324, 998-1000.

Woloshin, S., Patel, N. and Kesselheim, A. S. (2020) False negative tests for SARS-CoV-2 infection -challenges and implications. New England Journal of Medicine 383, e38.

Worldometer. (2020) COVID-19 Coronavirus Pandemic. Available at: https://www.worldometers.info/coronavirus/ (Accessed: December 8, 2020).

Zhang, J., McCullough, P. A. and Tecson, K. M. (2020) Vitamin D deficiency in association with endothelial dysfunction: Implications for patients with COVID-19. Reviews in Cardiovascular Medicine 21, 339344. 